\title{
An unusual bubble in the tidal arm of NGC 7318B in Stephan's Quintet
}

\author{
Y. Ohyama, S. Nishiura, T. Murayama, and Y. Taniguchi \\ Astronomical Institute, Tohoku University, \\ Aramaki, Aoba, Sendai 980-8578, JAPAN
}

\begin{abstract}
We optically find an unusual emission-line bubble near the tip of the southeastern arm of NGC 7318B. A large number $\left(\sim 10^{6}\right)$ of supernova remnants would be the most plausible agents.
\end{abstract}

NGC 7318B in Stephan's Quintet has two optical arms (toward N and s), emanating from the eastern part of the main body. Since these arms are similar morphologically to the tidal tails of merging galaxies such as NGC 4038/9, it is considered that NGC 7318B itself is a major merger with a retrograde orbit. Because the radio and the soft X-ray emission is associated to the arm (van der Hulst \& Rots 1981; Pietsch et al. 1997), it is of importancce to study its optical emission-line activity.

Our new CCD narrow-band $\mathrm{H} \alpha$ imaging shows a large-scale arc in $\mathrm{H} \alpha$ emission which traces closely the arms. This $\mathrm{H} \alpha$ arc resembles both the radio and the soft X-ray arcs morphologically, suggesting that a single physical mechanism is responsible for all these kinds of emission. Our optical spectroscopic observations of the shell-like feature at the southern tip of the arc reveal both broad $\mathrm{H} \alpha$ emission and stronger-than-normal [NII] and [SII] emission lines, which are typical of the supernova remnants. The required number of SNRs is estimated to be as large as $\sim 10^{6}$.

The proposed scenario for the arc formation is the following (Ohyama et al. 1997). The two tidal tails were formed during the past merging event between two gas-rich disk galaxies. Giant $\mathrm{H}$ II regions containing numerous massive stars $\left(\sim 10^{6}\right)$ were formed almost simultaneously along the tails (e.g., Barnes \& Hernquist 1992). After $\sim 10^{6-7}$ years, supernovae exploded almost simultaneously and formed the emission arc observed in $\mathrm{H} \alpha$, radio, and soft $\mathrm{X}$ ray.

\section{References}

Barnes, J.E., Hernquist, L. 1992, Nature 360, 715

van der Hulst, J.M., Rots, A.H. 1981, AJ 86, 1775

Ohyama, Y., Nishiura, S., Murayama, T., Taniguchi, Y. 1998, ApJ 492, L25

Pietsch, W., Trinchieri, G., Arp, H., Sulentic, J.W. 1997, A\&A 322, 89 